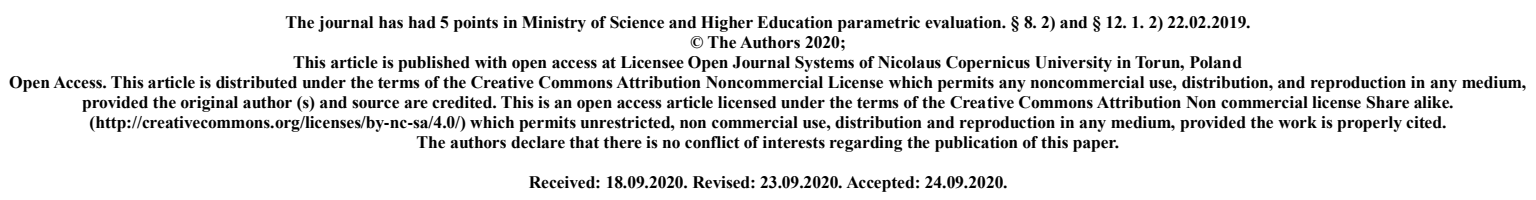

\title{
Chronic Fatigue Syndrome in children population - current knowledge summary
}

\author{
Monika Prylińska ${ }^{1}$, Natalia Skierkowska ${ }^{2}$, Weronika Topka ${ }^{2}$, Małgorzata Kwiatkowska ${ }^{2}$ \\ 1. Division of Ergonomics and Exercise Physiology, Department of Hygiene, \\ Epidemiology, Ergonomics and Postgraduate Education, Collegium Medicum in Bydgoszcz, \\ Nicolaus Copernicus University in Torun, Poland \\ 2. Faculty of Health Sciences, and the Department of Geriatrics Clinic, Collegium \\ Medicum in Bydgoszcz, Nicolaus Copernicus University in Torun, Poland
}

\begin{abstract}
Chronic Fatigue Syndrome/Myalgic Encephalomyelits (CFS/ME) is a chronic disease with complex pathophysiology and unknown etiology. It occurs both in children and adolescents, as well as in adults, with equal frequency. The clinical course is characterized by progressive fatigue, a significant reduction in the body's efficiency, lack of relief despite rest, and numerous accompanying symptoms. Pathognomonic symptom for PE is the increase in fatigue after physical or mental exertion and the persistence of these symptoms for several hours or days. The basis for effective treatment is primarily non-pharmacological treatment, including determining the optimal balance between physical activity and rest, which is aimed at preventing post-workout fatigue. Pain, insomnia, IO, and other symptoms can be treated with medication. So far, little research has been done on the diagnosis and treatment of this disease in children. This publication aims to summarize the knowledge currently available on the Chronic Fatigue Syndrome in children and adolescents.
\end{abstract}

Key words: Chronic Fatigue Syndrome, fatigue, children. 


\section{INTRODUCTION}

Chronic Fatigue Syndrome / Myalgic Encephalomyelitis (CFS / ME) is a chronic disease of complex pathophysiology and unknown etiology in children, adolescents and adults. Many doctors, including paediatricians, are familiar with CFS, but do not have detailed knowledge of its diagnosis and treatment. [1] The following article collects the most important information about CFS in paediatrics.

\section{MATERIAL AND METHODS}

The literature was reviewed using the Google Scholar and Pubmed databases. From the selected literature, publications were selected regarding the latest discoveries and guidelines for dealing with ICZM in the developmental age group.

\section{CHRONIC FATIGUE SYNDROME - DEFINITION}

Chronic Fatigue Syndrome was first defined in 1988. by the US Centers for Disease Control and Prevention (CDC) [2]. However, the revised and current diagnostic criteria for the disease were not presented until 1994. by Fukuda et al. [3]. They are presented in Table 1.

Tab. 1. The 1994 Centers for Disease Control and Prevention (CDC) case definition for Chronic Fatigue Syndrome (Fukuda's criteria). [3]

\begin{tabular}{|c|c|}
\hline 1. & $\begin{array}{l}\text { Major criteria: } \\
\text { - The individual has severe chronic fatigue for } 6 \text { or more consecutive } \\
\text { months that is not due to ongoing exertion or other medical conditions } \\
\text { associated with fatigues (these other conditions need to be ruled out by } \\
\text { a doctor after diagnostic tests have been conducted). } \\
\text { - The fatigue significantly interferes with daily activities and work. }\end{array}$ \\
\hline 2. & $\begin{array}{l}\text { Minor criteria: } \\
\text { - post-exertional malaise lasting more than } 24 \text { hours. } \\
\text { - Unrefreshing sleep. } \\
\text { - Significant impairment of short-term memory or concentration. } \\
\text { - Muscle pain. } \\
\text { - Pain in joints without swelling or redness. } \\
\text { - Headaches of a new type, pattern, or severity. } \\
\text { - Tender lymph nodes in the neck or armpit. } \\
\text { - A sore throat that is frequent or recurring. }\end{array}$ \\
\hline \multicolumn{2}{|r|}{$\begin{array}{l}\text { These symptoms persisted or recurred during } 6 \text { or more consecutive months of illness and } \\
\text { they cannot have first appeared before the fatigue. }\end{array}$} \\
\hline
\end{tabular}


The diagnosis of Chronic Fatigue Syndrome is possible only when the patient meets all the large criteria and the presence of at least 4 out of 8 symptoms that constitute the small criteria [4]. The diagnosis based on the above criteria is a purely clinical diagnosis based on clinical symptoms, after excluding any other diseases that may cause fatigue in the patient through a medical examination and additional laboratory tests $[3,5]$.

The clinical course of CFS is characterized by intense, chronic fatigue and a significant reduction in physical and cognitive functions. The main feature of this disease is general weakness and/or apathy, and worsening of symptoms after any mental or physical exertion. A characteristic feature of post-exercise fatigue (PEM) is that it persists for several hours or even days and does not subside despite sleep or rest. [1]

So far, no specific, separate diagnostic criteria have been formulated for children, therefore the same criteria are used for the diagnosis of CFS in the developmental age population as for adults. Due to a different clinical picture in children and a different impact on the quality of life and everyday functioning, this is an aspect that should be emphasized during further research on CFS.

Symptoms of ME/CFS may appear suddenly, develop gradually, or be mild with sudden exacerbations. In the medical history (medical history examination) concerning the period preceding the diagnosis, one can find the presence of symptoms typical of CFS, in the form of periods of exacerbation and remission. [1]

Symptoms of the disease may vary in severity, although all patients exhibit a significant limitation in terms of physical and cognitive performance. In the clinical picture in children, more often than in adults, pain symptoms (headaches, abdominal pain), sleep disorders (e.g. insomnia, excessive drowsiness or sleep without rest), orthostatic intolerance (OI) appear), hypersensitivity to drugs, odors, noise, light, disturbances in thermoregulation, or gastrointestinal symptoms (such as abdominal pain, nausea and anorexia). On the other hand, symptoms such as disorders of neurocognitive functions (memory and concentration disorders, reduced perceptiveness) or symptoms of sore throat and flu appear less frequently in the developmental age group. [6] It is also important to remember that children and adolescents more often than adults exhibit comorbid depression or anxiety symptoms. Oliver et al. in 2012. published a retrospective review in which they compared 131 cases of children and adolescents diagnosed with CFS and described that as many as 56\% of them had at least one comorbid disease (psychiatric, neurological, gastroenterological diseases, respiratory and musculoskeletal diseases were first mentioned), which may mask the symptoms of CFS. Therefore, it should be emphasized that each child with suspected CFS requires a multispecialist medical assessment so that no comorbidities are overlooked in the diagnosis. [7]

Children and adolescents with mild symptoms can attend school full-time or part-time, but with high absenteeism and limited opportunities to play sports and engage in extracurricular activities. Today ME/CFS is the most common cause of long-term school absences. [8-13] Children and adolescents with more severe ME/CFS symptoms use wheelchairs, stay at home or stay in bed. Children with the most severe symptoms may have difficulty even using home schooling. The overall quality of life of young ME/CFS patients is often worse than that of people with other chronic diseases such as diabetes, epilepsy and cystic fibrosis. [14] 


\section{EPIDEMIOLOGY}

The incidence of CFS is not precisely defined. Depending on the adopted diagnostic criteria and the degree of restrictiveness in their compliance, various sources give the frequency from $0.002 \%$ to $1 \%$ of the population [8]. The prevalence of ME/CFS in children and adolescents has been estimated at $0.1-0.5 \%$ in various studies. [9, 10] We know, however, that due to the lack of an unambiguous definition of this disease and the lack of clear criteria for its diagnosis, the actual number of patients with $\mathrm{CP}$ is certainly much greater.

\section{CAUSES AND PATHOPHYSIOLOGY}

The etiology of CFS/ME has not been established so far. Confirmed pathophysiological disorders indicate that it is a multiorgan disease of somatic origin, and not, as initially thought, psychological. [1]

Diversified pathophysiological disorders have become the basis for the formulation of various hypotheses about the etiology of the disease, including infectious agents, immune disorders, autoimmune processes, intestinal dysbiosis, oxidative stress, cardiovascular disorders, neuroendocrine, metabolic stress, brain dysfunction, toxin action, genetic susceptibility, abnormal gene expression and various combinations of these factors. [1]

Sometimes CFS occurs in children and adolescents after an acute infection, most often with Epstein-Barr virus (EBV). The criteria for the diagnosis of ME/CFS 6, 12 and 24 months after suffering from infectious mononucleosis confirmed by a positive Monospot test result were met by 13, 7 and $4 \%$ of adolescents, respectively. [20]

Patients with diagnosed CFS have been shown to have higher levels of IL-6, IL-8, IL$1 \beta$ and TNF- $\alpha$ [21-23]. Studies suggest that in patients with chronic fatigue there is a diffuse, low-grade chronic inflammation in the intestinal wall [24], which constantly activates the immune system and increases the production of pro-inflammatory cytokines. An important link between the immune system and the brain is the hypothalamic-pituitary-adrenal axis [25]. Activation of the immune system and increased levels of pro-inflammatory cytokines, through this axis, intensify the release of corticoliberin (CRH) from the hypothalamus, which activates the hypothalamic-pituitary-adrenal axis, increases the level of stress hormones in the body $[25,26]$ and in response to the systemic stress response causes dysfunction and intestinal inflammation [24].

The composition of the intestinal microflora, however, can be disturbed by a number of factors, including stress [27]. It has been proved that stress can reduce the size of the Bifidobacteria and Lactobacilli populations in the intestinal microflora [28]. It has been shown that bacteria from the Bifidobacteria group reduce the level of intestinal lipopolysaccharide (LPS) in mice [29]. This results in the inhibition of LPS-dependent activation of NK cells, which significantly reduces the level of proinflammatory cytokines and cyclooxygenase 2 (COX-2) [29]. Interestingly, stress (psychological or caused by physical exhaustion) is a well-proven risk factor for chronic fatigue syndrome [30].

Research shows that in people with IBS there are clear, measurable changes in the composition of the intestinal microflora, namely the level of Bifidobacteria is significantly reduced and the level of aerobic bacteria increases [31]. 
The determination of the composition of the intestinal microflora from stool samples of patients with chronic fatigue syndrome proved that Escherichia coli bacteria accounted for $92.3 \%$ of the aerobic microflora composition in the control group, compared to $49 \%$ in the group of patients with CFS. On the other hand, aerobic bacteria of the genus Enterococcus and Streptococcus were overrepresented in the microflora of patients with IBS [31]. Moreover, it has been shown that the greater the growth of Enterococcus bacteria in patients with PE, the more severe their cognitive impairment and neurological disorders, such as anxiety, nervousness, memory impairment, easy forgetfulness and confusion [31].

Myhill et al. Propose that the etiology of CFS is associated with damage to the mitochondria [32]. Mitochondria carry out intracellular aerobic respiration and produce energy in the form of ATP. Damage to the mitochondria contributes to a decrease in energy production and a reduction in the body's endurance to fatigue, as in chronic fatigue syndrome. The low level of ATP in the body causes the mitochondria to switch to anaerobic metabolism, thanks to which small amounts of ATP and lactic acid are produced in the process of glycolysis. This is common in patients with PE [33]. Excess lactic acid in the blood can contribute to muscle aches, feelings of heaviness and weakness, headaches and throat aches, and can also damage muscles and nerves. Patients with PE report that their symptoms increase during sports [34]. Exercise in these patients causes a build-up of oxidative stress and mitochondrial depletion, which weakens muscle function. Muscle tissue biopsies in patients with confirmed PE have shown abnormal structure and functioning of mitochondria in this group of patients [39]. Additionally, it has been proved that the degree of mitochondrial dysfunction is strongly related to the severity of the symptoms of chronic fatigue syndrome [32].

Dizziness is very common in children and adolescents with ME/CFS, and prolonged standing may aggravate symptoms such as fatigue, headache, nausea, and cognitive impairment (35-37).

A controlled study showed more frequent occurrence of postural tachycardia syndrome (POTS) and neutrally mediated hypotension (NMH) in these age groups. [36]

Further research is needed to determine the causes of autonomic disorders (NMH and POTS). Both of these syndromes can occur after suffering from infectious diseases or as secondary disorders as a result of autoimmune processes.

Compared to healthy subjects, children and adolescents with ME/CFS also suffer from other cardiovascular disorders, including delayed recovery of brain oxygenation after a short standing position [38] and exacerbation of cognitive impairment after orthostatic stress. [37]

Occasionally, OI-targeted treatment alleviates ME/CFS symptoms in both children and adolescents and adults.

The main hypotheses regarding the pathophysiological mechanisms of ME/CFS are not necessarily mutually exclusive. For example, infections and immune mechanisms can cause cardiovascular disorders, which in turn affect inflammation and the delivery of oxygen to cells. A detailed discussion of the possible pathophysiological mechanisms underlying ME/CFS symptoms is beyond the scope of this publication. [1] 


\section{TREATMENT}

ME/CFS influences the remodeling of the whole life of young people. This disease is associated with the need to deal with debilitating and debilitating symptoms, it affects the relationship in the family, causes absenteeism at school and loss of contact with peers. These losses can cause chaos and crisis. [1]

To date, no treatment or cure for ME/CFS has been developed. Therefore, the doctor should first of all:

- do no harm

- try to improve the patient's daily functioning

- gradually increase its activity

- alleviate the characteristic symptoms of the disease

- support the patient and his family. [1]

It is often necessary to educate school staff about ME/CFS and make them aware that it is a physical / organic disease, not a mental disorder. [1]

The variety of symptoms and comorbidities excludes the possibility of using one treatment strategy in all patients. The patient, family and physician should be aware of the triggers for symptoms and be prepared to implement several strategies before improving.

The therapeutic management includes:

- early diagnosis

- educating the patient, family and school staff about the disease

- identifying the main causes of post-exercise symptoms

- non-pharmacological and pharmacological symptomatic treatment

- providing the patient with guidance on activity, diet, maintaining social contacts and the possibility of continuing education

- monitoring the progress of treatment.

Establishing a diagnosis of ME/CFS, and thus confirming that the complaints have a specific disease cause, brings relief to the patient and the family. When explaining the essence of the disorders, it is necessary to emphasize the reality of the disease despite correct laboratory blood test results and to emphasize the possibility of improving the patient's functioning. Unambiguous determination of the diagnosis of the disease usually helps to reduce the patient's and his family's fears as to the cause of the ailment (e.g. explaining that it is not cancer and does not lead to quick death), justifies the cause of the ailment, which is of great psychological importance for further therapy, facilitates establishing a partnership doctor-patient relationship, enables the joint creation of a treatment plan, as well as assistance in obtaining, for example, education appropriate for a sick child. [6]

Treatment includes the use of non-pharmacological and pharmacological methods. Usually, in children, pharmacological treatment is introduced as a last resort, but in some ailments it is most effective. Many children and adolescents with ME/CFS respond to much lower doses than the standard medication, so treatment should be started at a low dose and gradually increased. [1] 


\section{PROGNOSIS}

It is believed that the prognosis of young CFS/ME patients is better than that of adults. So far, few clinical trials have been conducted with an adequate number of patients and with a sufficiently long follow-up period, but they have not confirmed that factors such as the severity of symptoms and age at onset could predict long-term treatment outcomes. [6]

In nearly 700 young patients included in the observational study, in whom symptoms 'relief' was found, the disease lasted on average 4-5 years (range 1-15 years). In $60 \%$ of patients, symptoms resolved after 5 years, and in $88 \%$ after 12 years. Approximately $1 / 3$ of patients, after the symptoms of the disease had subsided, admitted modifying their daily activities in order to maintain their well-being [39].

On the other hand, several other clinical studies with a follow-up of 2-13 years have shown that $20-48 \%$ of patients do not improve symptoms and even experience increased fatigue and physical impairment. [39, 40] Even in the case of complete relief of symptoms, many patients suffer from symptoms that are not observed in healthy subjects [40].

\section{SUMMARY}

Chronic Fatigue Syndrome is a serious, chronic disease that significantly impairs the daily functioning of the patient and reduces his quality of life. Despite the increasing number of scientific publications in the field of Chronic Fatigue Syndrome, its etiology and causal treatment have still not been established. Most of the conducted research concerns the adult patient population, which leads to a glaring gap in knowledge about the clinical course, diagnosis and treatment of pediatric patients. This monograph summarizes the currently available knowledge about CFS in the developmental age population and highlights the areas that require further research and further explanation. 


\section{REFERENCES}

1. $\quad$ Rowe, Peter C., et al. "Myalgic encephalomyelitis/chronic fatigue syndrome diagnosis and management in young people: a primer." Frontiers in pediatrics 5 (2017): 121.

2. Holmes, Gary P., et al. "Chronic fatigue syndrome: a working case definition." Annals of internal medicine 108.3 (1988): 387-389.

3. Fukuda, K., Straus, S. E., Hickie, I., Sharpe, M. C., Dobbins, J. G., Komaroff, A. "The chronic fatigue syndrome: a comprehensive approach to its definition and study." Annals of internal medicine 121.12 (1994): 953-959.

4. Kurowski, M., Kuna, P., "Zespót przewlektego zmęczenia." Alergia Astma Immunolo.

5. Carruthers, Bruce M., et al. "Myalgic encephalomyelitis: international consensus criteria." Journal of internal medicine 270.4 (2011): 327-338.

6. Shepherd, Ch., Chaudhuri, A., ME/CFS/PVFS: An exploration of the key clinical issues. ME Association, 2018.

7. Oliver, L. M., Patel, K. "Co-morbid conditions in children with chronic fatigue syndrome/myalgic encephalomyelitis (CFS/ME)-a retrospective case note review of a large cohort." Archives of Disease in Childhood 97.Suppl 1 (2012): A105-A105.

8. Nijhof, S.L., Maijer, K., Bleijenberg, G., et al. "Adolescent chronic fatigue syndrome: prevalence, incidence, and morbidity." Pediatrics 127.5 (2011): e1169-e1175.

9. Crawley, E.M., Emond, A.M., Sterne, J.A.C. "Unidentified Chronic Fatigue Syndrome/myalgic encephalomyelitis (CFS/ME) is a major cause of school absence: surveillance outcomes from school-based clinics." BMJ open 1.2 (2011): e000252.

10. Dowsett, E.G., Colby, J. "Long-term sickness absence due to ME/CFS in UK schools: an epidemiological study with medical and educational implications." Journal of Chronic Fatigue Syndrome 3.2 (1997): 29-42.

11. Crawley, E.M., Sterne, J.A. "Association between school absence and physical function in paediatric chronic fatigue syndrome/myalgic encephalopathy." Archives of Disease in Childhood 94.10 (2009): 752-756.

12. Smith, M.S., Martin-Herz, S.P., Womack, W.M., Marsigan, J.L. "Comparative study of anxiety, depression, somatization, functional disability, and illness attribution in adolescents with chronic fatigue or migraine." Pediatrics 111.4 (2003): e376-e381.

13. Kennedy, G., Underwood, C., Belch, J.J.F. "Physical and functional impact of chronic fatigue syndrome/myalgic encephalomyelitis in childhood." Pediatrics 125.6 (2010): e1324-e1330.

14. Winger, A., Kvarstein, G., Wyller, V.B., et al. "Health related quality of life in adolescents with chronic fatigue syndrome: a cross-sectional study." Health and Quality of Life Outcomes 13.1 (2015): 96.

15. Rimes, K.A., et al. "Incidence, prognosis, and risk factors for fatigue and chronic fatigue syndrome in adolescents: a prospective community study." Pediatrics 119.3 (2007): e603-e609.

16. Bombardier, C.H., Buchwald, D., "Chronic fatigue, chronic fatigue syndrome, and fibromyalgia: disability and health-care use." Medical care (1996): 924-930.

17. Knight, S., et al. "Paediatric chronic fatigue syndrome: complex presentations and protracted time to diagnosis." Journal of paediatrics and child health 49.11 (2013): 919-924.

18. Underhill, R.A., O'gorman, R. "Prevalence of chronic fatigue syndrome and chronic fatigue within families of CFS patients." Journal of Chronic Fatigue Syndrome 13.1 (2006): 3-13.

19. Buchwald D., Herrell R., Ashton S., et al "A twin study of chronic fatigue." Psychosomatic medicine 63.6 (2001): 936-943.

20. Barron D.F., Cohen B.A., Geraghty M.T., Violand R., Rowe P.C Barron, Diana F., et al. "Joint hypermobility is more common in children with chronic fatigue syndrome than in healthy controls." The Journal of pediatrics 141.3 (2002): 421-425.

21. Katz B.Z., Shiraishi Y., Mears C.J., Binns H.J., Taylor R., et al. "Chronic fatigue syndrome after infectious mononucleosis in adolescents." Pediatrics 124.1 (2009): 189-193.

22. Sulheim D.E., Fagermoen E., Winger A., et al.: "Disease mechanisms and clonidine treatment in adolescent chronic fatigue syndrome: a combined cross-sectional and randomized clinical trial." JAMA pediatrics 168.4 (2014): 351-360. 
23. Diaz-Mitoma F., Turgonyi E., Kumar A., et al. "Clinical improvement in chronic fatigue syndrome is associated with enhanced natural killer cell-mediated cytotoxicity: the results of a pilot study with Isoprinosine ${ }^{\circledR}$." Journal of Chronic Fatigue Syndrome 11.2 (2003): 71-95.

24. Scully, P., McKernan, D. P., Keohane, J., Groeger, D., Shanahan, F., Dinan, T. G., Quigley, E. M. "Plasma cytokine profiles in females with irritable bowel syndrome and extra-intestinal co-morbidity." American Journal of Gastroenterology 105.10 (2010): 2235-2243.

25. Clarke, G., Quigley, E. M., Cryan, J. F., Dinan, T. G. "Irritable bowel syndrome: towards biomarker identification." Trends in molecular medicine 15.10 (2009): 478-489.

26. Gaab, J., Rohleder, N., Heitz, V., Engert, V., Schad, T., Schürmeyer, T. H., Ehlert, U. "Stress-induced changes in LPS-induced pro-inflammatory cytokine production in chronic fatigue syndrome." Psychoneuroendocrinology 30.2 (2005): 188-198.

27. Raber, J., Sorg, O., Horn, T. F. W., Yu, N., Koob, G. F., Campbell, I. L., Bloom, F. E. "Inflammatory cytokines: putative regulators of neuronal and neuro-endocrine function." Brain research reviews 26.2-3 (1998): 320-326.

28. Jiménez, M. B. "Treatment of irritable bowel syndrome with probiotics. An etiopathogenic approach at last." Rev Esp Enferm Dig (Madrid) 101 (2009): 553-564.

29. Bailey, M. T., Lubach, G. R., \& Coe, C. L. "Prenatal stress alters bacterial colonization of the gut in infant monkeys." Journal of pediatric gastroenterology and nutrition 38.4 (2004): 414-421.

30. Riedel, C. U., Foata, F., Philippe, D., Adolfsson, O., Eikmanns, B. J., Blum, S. "Anti-inflammatory effects of bifidobacteria by inhibition of LPS-induced NF- $\kappa B$ activation." World journal of gastroenterology: WJG 12.23 (2006): 3729.

31. Maes, M., Mihaylova, I., Leunis, J. C. "Increased serum IgA and IgM against LPS of enterobacteria in chronic fatigue syndrome (CFS): indication for the involvement of gram-negative enterobacteria in the etiology of CFS and for the presence of an increased gut-intestinal permeability." Journal of affective disorders 99.1-3 (2007): 237-240.

32. Maes, M., Twisk, F. N. "Chronic fatigue syndrome: Harvey and Wessely's (bio) psychosocial model versus a bio (psychosocial) model based on inflammatory and oxidative and nitrosative stress pathways." BMC medicine 8.1 (2010): 35.

33. Myhill, S., Booth, N. E., McLaren-Howard, J. "Chronic fatigue syndrome and mitochondrial dysfunction." International journal of clinical and experimental medicine 2.1 (2009): 1.

34. Sheedy, J. R., Wettenhall, R. E., Scanlon, D., Gooley, P. R., Lewis, D. P., Mcgregor, N., De Meirleir, K. L. "Increased d-lactic acid intestinal bacteria in patients with chronic fatigue syndrome." In Vivo 23.4 (2009): 621-628.

35. Jammes, Y., Steinberg, J. G., Mambrini, O., Bregeon, F., Delliaux, S. "Chronic fatigue syndrome: assessment of increased oxidative stress and altered muscle excitability in response to incremental exercise." Journal of internal medicine 257.3 (2005): 299-310.

36. Rowe, P.C., Bou-Holaigh, I., Kan, J.S., Calkins, H. "Is neurally mediated hypotension an unrecognised cause of chronic fatigue?." The Lancet 345.8950 (1995): 623-624.

37. Stewart, J.M., Gewitz, M.H., Weldon, A., et al. "Orthostatic intolerance in adolescent chronic fatigue syndrome." Pediatrics 103.1 (1999): 116-121.

38. Stewart, J.M., Medow, M.S., Messer, Z.R., et al. "Postural neurocognitive and neuronal activated cerebral blood flow deficits in young chronic fatigue syndrome patients with postural tachycardia syndrome." American Journal of Physiology-Heart and Circulatory Physiology 302.5 (2012): H1185-H1194.

39. Rowe, K.S., Moon, J. "What is the natural history of chronic fatigue syndrome in young people? Oral presentation IACFS/ME conference Ottawa." Bull IACFS/ME Fall (2011): 129.

40. Bell, D.S., Jordan, K., Robinson, M. "Thirteen-year follow-up of children and adolescents with chronic fatigue syndrome." Pediatrics 107.5 (2001): 994-998. 